\title{
Incidence of Canine Glaucoma with Goniodysplasia in Japan : A Retrospective Study
}

\author{
Kumiko KATO ${ }^{1)}$, Nobuo SASAKI ${ }^{2) *}$, Satoru MATSUNAGA ${ }^{1)}$, Ryohei NISHIMURA ${ }^{2)}$ and Hiroyuki OGAWA ${ }^{1)}$ \\ ${ }^{1)}$ Laboratories of Veterinary Emergency Medicine and ${ }^{2)}$ Veterinary Surgery, Graduate School of Agricultural and Life Sciences, The \\ University of Tokyo, 1-1-1 Yayoi, Bunkyo-ku, Tokyo 113-8657, Japan
}

(Received 20 July 2005/Accepted 20 April 2006)

\begin{abstract}
The incidence of primary and secondary glaucoma in dogs was investigated. A total of 1244 dogs received ophthalmologic examinations, including tonometry and gonioscopy. Goniophotographs were taken using a goniolens to evaluate the iridocorneal angle (ICA) as well as pectinate ligament (PL). The anterior width of the ciliary cleft and the total distance from the origin of the PL to the anterior corneal surface were measured from the goniophotographs. Glaucoma was diagnosed based on the cupping of the optic nerve head, clinical signs, ocular changes, and high IOP, and it was synchronized with gonioscopic grades to differentiate between primary and secondary glaucoma. We investigated 1244 dogs of 29 breeds, including the mixed breed; among these, glaucoma was diagnosed in 127 dogs (162 eyes). Of 162 eyes, primary glaucoma was diagnosed in 129 eyes and secondary glaucoma in 33 eyes. Shiba Inu dogs (42 dogs, 33\%) showed the highest incidence of glaucoma, followed by Shih-Tzu (21 dogs, 16.5\%). Furthermore, all the glaucomatous Shiba Inu dogs had primary glaucoma with abnormal ICA grades and dysplastic PLs. The findings of our study reveal that the Shiba Inu breed in Japan may have a hereditary predisposition to glaucoma.

KEY WORDS: canine, glaucoma, gonioscopy, incidence, ophthalmologic examination.
\end{abstract}

J. Vet. Med. Sci. 68(8): 853-858, 2006

Canine glaucoma is generally classified as either primary or secondary. In primary glaucoma, intraocular pressure (IOP) increases through a reduction in aqueous fluid drainage due to hereditary abnormality, which is not associated with other ocular diseases. Primary glaucoma has bilateral potential; however, the time of onset in the two eyes may show considerable variation. It has been considered that primary glaucoma is inherited in Beagles [8, 10, 13, 14] and Welsh Springer Spaniels [5], while hereditary components have been suggested in several other breeds $[3,19,22]$. The most frequent causes of secondary glaucoma include lens displacement, anterior uveitis, intraocular neoplasia, postcataract surgery, and trauma [9]. The mechanism behind the elevated IOP is generally assumed to be the obstruction of the aqueous humor outflow pathways [9].

Various techniques have been reported to identify primary glaucoma in several breeds $[2,4,6,7]$. Gonioscopy is the classical and standard method for the qualitative evaluation of the iridocorneal angle (ICA) width and anatomy and the degree of pectinate ligament (PL) dysplasia [27]. The anterior portion of the ICA can be easily viewed by gonioscopy [2]. Ekesten and Narfrström [7] investigated the objective measuring method for angle width in Samoyeds. This method was modified and applied to other breeds in two studies $[4,6]$. Recent reports have shown that the efficacy of ultrasound biomicroscopy [15] and A-mode ultrasound scanning in comparison with gonioscopy for the measurement of ICA and anterior chamber depth in dogs [28].

Gelatt and Mackay [11] investigated the prevalence of

\footnotetext{
* Correspondence to: Dr. SASAKi, N., Laboratory of Veterinary Surgery, Graduate School of Agricultural and Life Sciences, The Univesity of Tokyo, 1-1-1 Yayoi, Bunkyo-ku, Tokyo 113-8657, Japan.
}

breed-related glaucoma in pure-bred dogs. They also reported the prevalence of glaucoma in newer breeds, including Chow Chow, Australian Cattle dog, Shar-Pei, Bichon Frise, Akita, Jack Russell Terrier, and Bouvier des Flandres that have become popular in North America over the past 20 years. We previously reported the possible association of glaucoma with PL dysplasia and narrowing of the ICA in Shiba Inu dogs in Japan [17].

The purpose of this study was to investigate the incidence of canine glaucoma in various breeds of dogs in Japan, including Shiba Inu dogs, and to identify the involvement of ICA grade with glaucoma in these breeds.

\section{MATERIALS AND METHODS}

The study was carried out from October 1999 to May 2004; 1244 dogs were investigated by ophthalmologic examinations in the Veterinary Medical Center (VMC), The University of Tokyo. These dogs were referred for eye diseases, neurological diseases, or other diseases. During the same time period, 8103 dogs of various breeds visited VMC with other problems.

All the dogs received complete ophthalmologic examination using a hand-held slit-lamp biomicroscope (SL-14; Kowa, Tokyo, Japan) as well as indirect ophthalmoscopy (IO- $\alpha$ TVII; Neitz, Tokyo, Japan), tonometry (Tono-Pen XL; Mentor ${ }^{\circledR}$ O\&O, Inc., Norwell, MA, U.S.A.), and gonioscopy (OLI-1 Layden Infant Lens, $11.5 \mathrm{~mm}$; Koeppe, $17.0 \mathrm{~mm}$; Ocular Instruments Inc., Bellevue, WA, U.S.A.). We used two types of gonioscopic lenses based on the palpebral fissures of dogs; most Shiba Inu dogs had narrow palpebral fissures and small eyeballs as compared to other breeds [1]. Photographs taken through the goniolens of a fundus camera (Genesis, Kowa, Tokyo) were used to evalu- 
ate ICA as well as PL. These procedures were performed in the conscious state with topical anesthesia (oxybuprocaine hydrochloride; Santen, Oosaka, Japan) for corneal and conjunctival surfaces.

The ICA and PL were measured by using Ekesten's method [7] with a modification. The ICA was evaluated over an area of approximately $270^{\circ}$, from the $2 o^{\prime}$ clock to 10 o'clock position (nasal-ventral-temporal). The PLs of individual dogs were documented by photographs taken through the goniolens. The anterior width of the ciliary cleft and the total distance from the origin of the PLs to the anterior corneal surface were measured from the goniophotographs. The ratios of the width of the PL to that of the anterior corneal surface (often the anterior border of the superficial pigmented zone) were classified into 4 grades based on the classification by Ekesten and Narfrström [7]: closed (ratio $\leq 0.15)$, narrow (0.15-0.3), slightly narrow $(0.3-0.45)$, and open $(0.45-0.55)$. Their classification also included the grade "wide open" (ratio $\geq 0.55$ ); however, in this study, none of the dogs fulfilled the criteria for the "wide open" grade.

Glaucoma was diagnosed based on the following abnormal findings: cupping of the optic nerve head, visual disturbance or loss of vision, ocular abnormalities such as conjunctival hyperemia, corneal striae, corneal edema, buphthalmos, and high IOP (more than $25 \mathrm{mmHg}$ ). The diagnosis of glaucoma was confirmed in patients with two or more of the abnormal findings described above. In the case of patients with only one abnormal finding, other clinical symptoms such as mydriasis, blepharospasm, and the rubbing behavior in the eye or periocular tissues were assessed and glaucoma was diagnosed. Primary glaucoma was defined as the increased level of IOP that is detrimental to the maintenance of vision and health of the eye, without other intraocular diseases [9]. When the evaluation of ICA and PL was difficult due to the loss of transparency of the cornea, the contralateral eye was assessed. The decision of classifying glaucoma as primary or secondary was made based on the clinical history of trauma or other ocular problems and was synchronized with gonioscopic grades of the contralateral eye. Among 5 dogs (4 Shiba Inu dogs and an American Cocker Spaniel) had already bilateral buphthalmos, and hence, we could not evaluate ICA and PL at the first admission. Those dogs were excluded from this study.

Statistical analysis was performed using the Fisher's exact probability test to prevent gender bias between primary glaucoma and secondary glaucoma in each breed. A Steel-Dwass of a post-hoc test was used to evaluate the incidence of glaucomatous dogs among the total dogs of the same breed and dogs receiving ophthalmologic examinations. Statistical significance was defined at $P<0.05$.

\section{RESULTS}

Among 1244 dogs of 71 breeds receiving complete ophthalmologic examination, Shih-Tzus (218), Shiba Inu dogs (145), Miniature Dachshunds (96), Mixed breed dogs (95),
Yorkshire Terrier (66), Malteses (64), Golden Retrievers (56), Chihuahua (47), Pugs (42), Cavalier King Charles Spaniels (41), Pomeranians (37), Shetland Sheepdogs (36), American Cocker Spaniels (33), Toy Poodles (31), Labrador Retrievers (27), Beagles (24), Miniature Schnauzers (20), Papillons (20), Welsh Corgis (14), West Highland White Terriers (12) were the more common breeds. Other breeds included less than 10 dogs.

Glaucoma was diagnosed in 127 dogs of 29 breeds (162 eyes) (Table 1). These breeds included Shiba Inu dogs (42), Shih-Tzus (21), mixed breed dogs (10), American Cocker Spaniels (8), Beagles (5), Golden Retrievers (5), Toy Poodles (4), Malteses (3), Miniature Dachshunds (3), Yorkshire Terriers (3), Cavalier King Charles Spaniels (2), Papillons (2), Pugs (2), Welsh Corgis (2), and one each of the following breeds: Ainu (Hokkaido) Inu dog, Akita, Basset Hound, Belgian Tervuren, Bull Terrier, Fox Terrier, French Bulldog, Japanese Chin, Labrador Retriever, Old English Sheepdog, Pekingese, Pitbull Terrier, Pomeranian, Samoyed, and Tibetan Terrier.

Among 127 glaucomatous dogs, 33\% (42/127) were Shiba Inu dogs and 16.5\% (21/127) were Shih-Tzus, followed by mixed breeds (7.9\%: 10/127), American Cocker Spaniels (6.3\%: 8/127), Beagles, and Golden Retrievers (3.9\%: 5/127), Toy Poodles (3.1\%: 4/127), Malteses, Miniature Dachshunds, and Yorkshire Terriers (2.4\%: 2/127), and others.

Among the 162 glaucomatous eyes, primary glaucoma was diagnosed in 129 eyes and secondary glaucoma in 33 eyes. Primary glaucoma with abnormal ICA grades was diagnosed in all the glaucomatous eyes (58 eyes) of 42 Shiba Inu dogs. Bilateral glaucoma was observed in 16 Shiba Inu dogs. In Shih-Tzus, primary glaucoma with abnormal ICA was diagnosed in 15 glaucomatous eyes (14 dogs); however, ICA grading of Shih-Tzus was less severe than that of Shiba Inu dogs. Secondary glaucoma was diagnosed in the remaining eight glaucomatous eyes (34.8\%) of Shih-Tzus; among these, glaucoma may have occurred in three eyes because of retinal detachment and vitreous degeneration. In American Cocker Spaniels, primary glaucoma with abnormal ICA grades was diagnosed in seven eyes of eight dogs. In two eyes with secondary glaucoma, the cause could have been lens- or cataract- induced uveitis, although the eyes showed abnormal ICA grades. In Beagles, primary glaucoma was diagnosed in three eyes of three dogs; one dog had open ICA, while the remaining two dogs had closed or narrow ICA. In Golden Retrievers, secondary glaucoma induced by uveitis was diagnosed in five dogs; two dogs had narrow ICA, and the remaining three had open ICA. Among other breeds with less than five glaucomatous dogs, primary glaucoma with abnormal ICA was diagnosed in Miniature Dachshunds.

ICAs of 103 non-glaucomatous Shiba Inu dogs were closed in 12 , narrow in 30 , slightly narrow in 41 , and open in 20 dogs. Similarly, ICAs of 197 non-glaucomatous ShihTzus were closed in 28 , narrow in 42 , slightly narrow in 75 , and open in 52 dogs. ICAs of 25 non-glaucomatous Ameri- 
Table 1. The incidence of canine glaucoma in various breeds in Japan with regard to gender, age of onset, type of glaucoma, and ICA grades

\begin{tabular}{|c|c|c|c|c|c|c|c|c|c|c|c|c|c|}
\hline \multirow{4}{*}{ Breeds } & \multicolumn{3}{|c|}{ Age of onset (years) } & \multicolumn{10}{|c|}{ Glaucomatous eyes } \\
\hline & \multirow{3}{*}{$\begin{array}{c}\text { Number of } \\
\text { dogs with } \\
\text { glaucoma } \\
(\%)\end{array}$} & \multirow[t]{3}{*}{ Male/Female } & \multirow[t]{3}{*}{$\mathrm{M} \pm \mathrm{SD}$ (Range) } & \multirow{2}{*}{\multicolumn{4}{|c|}{$\begin{array}{c}\text { Primary } \\
\text { ICA grade }\end{array}$}} & \multirow{2}{*}{\multicolumn{4}{|c|}{$\begin{array}{l}\text { Secondary } \\
\text { ICA grade }\end{array}$}} & \multirow{2}{*}{\multicolumn{2}{|c|}{$\begin{array}{l}\text { Total } \\
\text { eyes }\end{array}$}} \\
\hline & & & & & & & & & & & & & \\
\hline & & & & $\mathrm{C}$ & $\mathrm{N}$ & SN & $\mathrm{O}$ & $\mathrm{C}$ & $\mathrm{N}$ & SN & $\mathrm{O}$ & $P$ & $\mathrm{~S}$ \\
\hline Shiba Inu dog & $42(33)$ & $20 / 22$ & $8.4 \pm 3.1(3.1-14)$ & 38 & 15 & 5 & - & - & - & - & - & 58 & - \\
\hline Shih-Tzu & $21(16.5)$ & $10 / 11$ & $8.3 \pm 2.1(4-11)$ & 5 & 9 & 1 & - & - & - & 3 & 5 & 15 & 8 \\
\hline Mixed breed & $10(7.9)$ & $4 / 6$ & $8.1 \pm 3.1(2-13)$ & 5 & 3 & - & 1 & - & - & - & 3 & 9 & 3 \\
\hline American Cocker Spaniel & $8(6.3)$ & $4 / 4$ & $7.4 \pm 2.5(4-11)$ & 3 & 3 & 1 & - & - & 2 & - & - & 7 & 2 \\
\hline Beagle & $5(3.9)$ & $3 / 2$ & $6.8 \pm 2.5(5-11)$ & 2 & 2 & - & 2 & - & - & - & 3 & 6 & 3 \\
\hline Golden Retriever & $5(3.9)$ & $3 / 2$ & $11.4 \pm 0.8(10-12)$ & - & - & - & - & - & 2 & - & 3 & - & 5 \\
\hline Toy Poodle & $4(3.1)$ & $3 / 1$ & $11.8 \pm 5.1(6-18)$ & 2 & 1 & - & - & - & - & - & 1 & 3 & 1 \\
\hline Maltese & $3(2.4)$ & $2 / 1$ & $9.7 \pm 5.9(3-14)$ & - & 1 & - & - & - & - & 2 & 1 & 2 & 3 \\
\hline Miniature Dachshund & $3(2.4)$ & $1 / 2$ & $3.7 \pm 3.1(1-7)$ & 1 & 2 & - & - & - & - & - & - & 3 & - \\
\hline Yorkshire Terrier & $3(2.4)$ & $-/ 3$ & $6.7 \pm 1.5(5-8)$ & - & 1 & 1 & - & - & - & - & 1 & 2 & 1 \\
\hline Cavalier King Charles Spaniel & $12(1.6)$ & $-/ 2$ & $11.5(10-13)$ & 1 & - & 1 & - & - & - & - & - & 2 & - \\
\hline Papillon* & $2(1.6)$ & $-/ 2$ & $5.5(4-7)$ & 1 & 1 & - & - & - & - & - & - & 2 & - \\
\hline Pug & $2(1.6)$ & $1 / 1$ & $5.5(5-6)$ & - & 1 & - & - & - & - & - & 1 & 1 & 1 \\
\hline Welsh Corgi & $2(1.6)$ & $2 /-$ & $6(6)$ & - & 1 & 1 & - & - & - & - & - & 2 & - \\
\hline Ainu (Hokkaido) Inu dog* & $1(0.8)$ & $1 /-$ & 10 & 2 & - & - & - & - & - & - & - & 2 & - \\
\hline Akita & $1(0.8)$ & $1 /-$ & 2 & - & - & - & - & - & - & 2 & - & - & 2 \\
\hline Basset Hound & $1(0.8)$ & $-/ 1$ & 8 & 1 & - & - & - & - & - & - & - & 1 & - \\
\hline Belgian Tervuren & $1(0.8)$ & $-/ 1$ & 11 & 2 & - & - & - & - & - & - & - & 2 & - \\
\hline Bull Terrier* & $1(0.8)$ & $-/ 1$ & 4 & 2 & - & - & - & - & - & - & - & 2 & - \\
\hline Fox Terrier & $1(0.8)$ & $-/ 1$ & 8 & 2 & - & - & - & - & - & - & - & 2 & - \\
\hline French Bulldog* & $1(0.8)$ & $-/ 1$ & 2 & 2 & - & - & - & - & - & - & - & 2 & - \\
\hline Japanese Chin & $1(0.8)$ & $-/ 1$ & 1 & - & - & - & - & - & - & - & 2 & - & 2 \\
\hline Labrador Retriever & $1(0.8)$ & $-/ 1$ & 10 & 2 & - & - & - & - & - & - & - & 2 & - \\
\hline Old English Sheepdog* & $1(0.8)$ & -11 & 14 & - & 2 & - & - & - & - & - & - & 2 & - \\
\hline Pekingese & $1(0.8)$ & $-/ 1$ & 7 & - & - & - & - & - & - & 1 & - & - & 1 \\
\hline Pitbull Terrier & $1(0.8)$ & $-/ 1$ & 12 & - & - & - & - & - & - & - & 2 & - & 2 \\
\hline Pomeranian* & $1(0.8)$ & $-/ 1$ & 12 & 2 & - & - & - & - & - & - & - & 2 & - \\
\hline Samoyed & $1(0.8)$ & $1 /-$ & 6 & 2 & - & - & - & - & - & - & - & 2 & - \\
\hline Tibetan Terrier & $1(0.8)$ & $-/ 1$ & 1 & - & 2 & - & - & - & - & - & - & 2 & - \\
\hline Total & 127 & $56 / 71$ & & 74 & 43 & 10 & 2 & 0 & 4 & 8 & 21 & 129 & 33 \\
\hline
\end{tabular}

$\mathrm{M} \pm \mathrm{SD}$ : mean of age \pm standard deviation.

ICA grades: C, Closed; N, Narrow; SN, Slightly Narrow; O, Open.

$\mathrm{P}$, Primary glaucomatous eyes; $\mathrm{S}$, Secondary glaucomatous eyes.

*: There was no previous report on primary glaucoma in these breeds.

can Cocker Spaniels were closed in 3, narrow in 3, slightly narrow in 11 , and open in 8 dogs.

With regard to gender, no statistically significant difference was observed between primary and secondary glaucoma in any of the breeds. However, the male patients were slightly more in number than the females in Beagles, Golden Retrievers, Toy Poodles, and Malteses. In contrast, the female patients were more in number than the males in Shiba Inu dogs, Shih-Tzus, Miniature Dachshunds, and mixed breed dogs.

The mean age of the glaucomatous dogs was more than the middle age in almost all the breeds, with the exception of Miniature Dachshunds, Papillons, and Pugs whose mean ages were $3.7 \pm 3.1,5.5 \pm 2.1$, and $5.5 \pm 0.7$ years, respectively.

Table 2 shows the number of dogs of each breed that visited VMC during the study period and the number of dogs with and without glaucoma.
Among 8103 dogs included in this study, 10.1\% (818 dogs) were Miniature Dachshunds, 8.4\% (681 dogs) were Shih-Tzus, $8.2 \%$ (662 dogs) were Golden Retrievers, 4.8\% (386 dogs) were Yorkshire Terriers, 3.6\% (288 dogs) were Malteses, 3.4\% (275 dogs) were Beagles, 2.9\% (238 dogs) were Shiba Inu dogs, 2.7\% (216 dogs) were Toy Poodles, and 1.4\% (114 dogs) were American Cocker Spaniels. Among the dogs of same breed receiving ophthalmologic examinations, the percentage of glaucomatous Shiba Inu dogs was higher $(3.4 \%)$ than that of other breeds; however, the difference was not statistically significant. A comparison of the number of glaucomatous dogs to the total number of dogs of the same breed showed that the Shiba Inu breed had highest percentage (17.6\%) of glaucomatous patients. In contrast, the percentages of glaucomatous patients among American Cocker Spaniels and Shih-Tzus were 7\% and $3.1 \%$, respectively. In other breeds such as Toy Poodles, Beagles, Malteses, Golden Retrievers, and Yorkshire Terri- 
Table 2. The incidence of canine glaucoma in each breeds among the number of dogs of each breed visited to VMC during the study period

\begin{tabular}{lcccc}
\hline Breeds & $\begin{array}{c}\text { Number and percentages } \\
\text { of dogs of each breed } \\
\text { among the total number } \\
\text { of dogs visited VMC } \\
(8103 \text { dogs) }\end{array}$ & $\begin{array}{c}\text { Number and percentages of } \\
\text { glaucomatous dogs of each } \\
\text { breed among the total } \\
\text { number of dogs receiving } \\
\text { ophthalmologic } \\
\text { examinations (1244 dogs } *)\end{array}$ & $\begin{array}{c}\text { Percentages of primary and } \\
\text { secondary glaucomatous } \\
\text { dogs among the total number } \\
\text { of glaucomatous dogs in } \\
\text { each breed }\end{array}$ \\
\hline Shiba Inu dog & $(\%)$ & $(\%)$ & primary(\%) & secondary(\%) \\
Shih-Tzu & $238(2.9)$ & $42(3.4)$ & 17.6 & - \\
American Cocker Spaniel & $681(8.4)$ & $21(1.7)$ & 2.1 & 1 \\
Beagle & $114(1.4)$ & $8(0.6)$ & 5.3 & 1.8 \\
Golden Retriever & $275(3.4)$ & $5(0.4)$ & - & 0.7 \\
Toy Poodle & $662(8.2)$ & $5(0.4)$ & 1.4 & 0.8 \\
Maltese & $216(2.7)$ & $4(0.3)$ & 0.3 & 0.5 \\
Miniature Dachshund & $288(3.6)$ & $3(0.2)$ & 0.4 & - \\
Yorkshire Terrier & $818(10.1)$ & $3(0.2)$ & 0.5 & 0.3 \\
\hline
\end{tabular}

VMC: Veterinary Medical Center

*: The number of dogs showed those visited VMC during the study period.

ers, the percentages of glaucomatous dogs were less than $2 \%$. Although the percentage of Shiba Inu dogs with glaucoma was much higher than that of other breeds, the difference was not statistically significant.

\section{DISCUSSION}

This study revealed that primary glaucoma with abnormal ICA grades is more common in Shiba Inu dogs and ShihTzus, particularly in Shiba Inu dogs in Japan. Among dogs of various breeds that visited VMC during the study period, Shiba Inu dog was the seventh breed among the nine common breeds, whereas Shih-Tzu dog was the second common breed. Among the total number of patients in the same breed, the percentages of glaucomatous dogs were $17.6 \%$ in Shiba Inu dogs and $3.1 \%$ in Shih-Tzus. Additionally, among the total number of dogs of the same breed receiving ophthalmologic examination, the percentage of glaucomatous Shiba Inu dogs was 3.4\%; a similar incidence of primary glaucoma in Basset Hounds, American Cocker Spaniels, Welsh Terriers, and Fox Terriers was reported in North America during the years 1974-1993 [11]. Shih-Tzus accounted for $16.5 \%$ of the total glaucomatous dogs; however, among the total dogs with ophthalmic disorders (1244 dogs), the percentage of glaucomatous Shih-Tzus was 1.7\%. In addition, primary glaucoma was diagnosed in only 14 dogs of the Shih-Tzu breed, which accounted for $1.1 \%$ of the total number of dogs with eye disorders; a similar incidence was reported in Shih-Tzus in North America (19841993) [11].

Gelatt and Mackay [12] have reported that hyphema and secondary glaucoma were common in Shih-Tzu (19942003). In this study, $37.5 \%$ of Shih-Tzu patients had retinal detachment and secondary glaucoma. In American Cocker Spaniels, two dogs had secondary glaucoma that was caused by lens- or cataract-induced uveitis; a similar finding was reported by van der Woerdt et al. [26] and Gelatt and
Mackay [12]. In American Cocker Spaniels, the percentage of glaucomatous dogs among the total number of dogs of this breed that visited VMC during the study period was 7\%. This was the second highest incidence in the present study, and the percentage was almost similar to that reported in the previous studies $[11,19]$.

In Beagles, primary open-angle glaucoma has been investigated in previous studies $[8,13,14]$. In our study, three dogs of the Beagle breed had primary glaucoma, one dog had primary open angle glaucoma, and two other dogs had primary glaucoma with abnormal ICA. The glaucomatous onset occurred at the middle to older age, which was similar to that reported in the previous studies [11].

Some reports suggest a high prevalence of pigmentary uveitis-induced glaucoma in Golden Retrievers [12, 24]. However, in our study, the cause of uveitis-induced glaucoma in Golden Retrievers was unclear.

In our study, other breeds in which more than three glaucomatous patients were found included Maltese, Miniature Dachshund, and Yorkshire Terrier. Three Malteses had secondary glaucoma of unknown causes; however, it may be related to intraocular inflammation, as reported by Gelatt et al. [12]. In contrast, three Miniature Dachshund patients had primary glaucoma with abnormal ICA. Thus far, primary glaucoma has not been reported in Miniature Dachshund, and hence, further investigations are required. In Yorkshire Terrier, both primary and secondary glaucomas have been reported $[16,18]$. In our study, two primary and one secondary glaucomatous patients of Yorkshire Terrier breed were found.

Furthermore, although the tendency of male or female predominance was observed depending on the breed, the difference in the number of male and female patients was not statistically significant. Gender difference has been described with regard to pectinate ligament dysplasia (PLD) in some breeds, including American Cocker Spaniel [19], Welsh Springer Spaniel [5], and Siberian Husky, with 
females being more frequently affected. Bjerkås also reported that there may be breed-specific gender differences based on the clinical data [4].

We found that the onset of primary glaucoma occurred at the middle age, which was almost similar to that reported in previous studies $[4,9,11,21]$. It is believed that the impediment to the outflow facility at the level of the pupil may be associated with a relatively anteriorly positioned lens and increased axial length of the lens [7]. Moreover, the lenses of middle-aged or older dogs undergo a process of slow thickening since the time of birth [23]. This phenomenon may be related to the report that primary closed-angle glaucoma is at least eight times more common than primary open-angle glaucoma in dogs [23]. In addition, in closedangle glaucoma, the ICA appears narrow or partially closed on gonioscopy, or dysplastic pectinate ligaments are initially involved, leading to completely closed angle when the IOP is elevated [5, 7, 19, 20, 25, 29].

The age of onset of glaucoma in Miniature Dachshund was less than that of the other breeds. Glaucoma was diagnosed in two Pug dogs that were younger (5 years and 6 years) than the other glaucomatous dogs belonging to the other breeds. Gelatt and Mackay [11] reported that the age of onset of glaucoma in Pug was 5.4 years; this was similar to the age of onset observed for Pug dogs in the present study. There have been no previous reports on primary glaucoma in Miniature Dachshund.

In conclusion, Shiba Inu dogs in Japan are highly predisposed to primary glaucoma. The incidence of glaucoma in other breeds was almost similar to that previously reported. Further research should be conducted in order to investigate the hereditary and genetic background of glaucoma in Shiba Inu dogs.

ACKNOWLEDGEMENT. The authors would like to thank Dr. Alexandra van der Woerdt of the Animal Medical Center, New York, U.S.A., for her valuable advice and critical reading of this manuscript.

\section{REFERENCES}

1. American College of Veterinary Ophthalmologists Genetics Committee. 1993. In: Addendum to: Ocular Disorders Proven or Suspected to be Hereditary in Dogs, Canine Eye Registration Foundation, West Lafayette.

2. Bedford, P. G. C. 1973. A practical method of gonioscopy and goniophotography in the dog and cat. J. Small Anim. Pract. 14: 601-606.

3. Bedford, P. G. C. 1975. The aetiology of primary glaucoma in the dog. J. Small Anim. Pract. 16: 217-239.

4. Bjerkås, E., Ekesten, B. and Farstad, W. 2002. Pectinate ligament dysplasia and narrowing of the iridocorneal angle associated with glaucoma in the English Springer Spaniel. Vet. Ophthalmol. 5: 49-54.

5. Cottrell, B. D. and Barnett, K. C. 1988. Primary glaucoma in the Welsh Springer Spaniel. J. Small Anim. Pract. 29: 185199.

6. Ekesten, B., Bjerkås, E., Kongestengen, K. and Narfström, K. 1997. Primary glaucoma in the Norwegian Elkhound. Vet.
Comp. Ophthalmol. 7: 14-18.

7. Ekesten, B. and Narfström, K. 1991. Correlation of morphologic features of the iridocorneal angle to intraocular pressure in Samoyed. Am. J. Vet. Res. 52: 1875-1878.

8. Gelatt, K. N. 1972. Familial glaucoma in the beagle dog. J. Am. Anim. Hosp. Assoc. 8: 23-28.

9. Gelatt, K. N. and Brooks, D. E. 1999. The canine glaucomas. pp. 701-749. In: Veterinary Ophthalmology, 3rd ed. (Gelatt, K. N. ed.), Lippincott Williams \& Wilkins, Philadelphia.

10. Gelatt, K. N. and Gum, G. G. 1981. Inheritance of glaucoma in the beagle. Am. J. Vet. Res. 42: 1691-1693.

11. Gelatt, K. N. and Mackay, E. O. 2004. Prevalence of the breedrelated glaucomas in pure-bred dogs in North America. Vet. Ophthalmol. 7: 97-111.

12. Gelatt, K. N. and Mackay, E. O. 2004. Secondary glaucomas in the dog in north America. Vet. Ophthalmol. 7: 245-259.

13. Gelatt, K. N., Peiffer, R. L. Jr., Gwin, R. M., Gum, G. G. and Williams, L. W. 1977. Clinical manifestations of inherited glaucoma in the beagle. Invest. Ophthalmol. Vis. Sci. 16: 11351148.

14. Gelatt, K. N., Perffer, R. L. Jr., Gwin, R. M. and Saunk, J. J. Jr. 1976. Glaucoma in the Beagle. Tr. Am. Acad. Ophthalmol. Otol. 8: 634-644.

15. Gibson, T. E., Roberts, S. M., Severin, G. A. and Steyn, P. F. and Wrigley, R. H. 1998. Comparison of gonioscopy and ultrasound biomicroscopy for evaluating the iridocorneal angle in dogs. J. Am. Vet. Med. Assoc. 213: 635-638.

16. Hasegawa, T., Doki, K. and Yanase, J. 2001. Long-term management of a glaucomatous eye in a dog treated with medical therapy alone. J. Vet. Med. Sci. 63: 1323-1325.

17. Kato, K., Sasaki, N., Matsunaga, S., Mochizuki, M., Nishimura, R. and Ogawa, H. 2005. Possible association of glaucoma with pectinate ligament dysplasia and narrowing of the iridocorneal angle in Shiba Inu dogs in Japan. Vet. Ophthalmol. 9: 71-75.

18. Lin, C., Chou, Y. and Fei A. 2003. A retrospective study on clinical characteristics of canine uveitis. Taiwan Vet. J. 29: 200-204.

19. Lovekin, L. G. 1964. Primary glaucoma in dogs. J. Am. Vet. Med. Assoc. 145: 1081-1091.

20. Magrane, W.G. 1957. Canine glaucoma II. Primary classification. J. Am. Vet. Med. Assoc. 131: 372-374.

21. Martin, C. L. 1974. Development of pectinate ligament structure in the dog: study by scanning electron microscopy. Am. J. Vet. Res. 35: 1433-1439.

22. Martin, C. L. and Wyman, M. 1978. Primary glaucoma in the dog. Vet. Clin. North Am. 8: 257-286.

23. Miller, P. E., Schmidt, G. M., Vainisi, S. J., Swanson, J. F. and Herrmann, M. K. 2000. The efficacy of topical prophylactic antiglaucoma therapy in primary closed angle glaucoma in dogs: A multicenter clinical trial. J. Am. Anim. Hosp. Assoc. 36: $431-438$.

24. Sapienza, J. S., Simó Domenech, F. J. and Prades-Sapienza, A. 2000. Golden Retriever uveitis: 75 cases (1994-1999). Vet. Ophthalmol. 3: 241-246.

25. van der Linde-Sipman, J. S. 1987. Dysplasia of the pectinate ligaments and primary glaucoma in the Bouvier des Flandres dogs. Vet. Pathol. 24: 201-206.

26. van der Woerdt, A., Nasisse, M. P. and Davidson, M. G. 1992. Lens-induced uveitis in dogs: 151 cases (1985-1990). $J$. Am.Vet. Med. Assoc. 201: 921-926.

27. van Herick, W., Shaffer, R, N. and Schwartz, A. 1969. Estimation of width of angle of anterior chamber. Am. J. Ophthalmol. 
68: 626-629.

28. Wood, J. L. N., Lakhani, K. H., Mason, I. K. and Barnett, K. C. 2001. Relationship of the degree of goniodysgenesis and other ocular measurements to glaucoma in Great Danes. Am. J. Vet.
Res. 62: 1493-1499.

29. Wyman, M. and Ketring, K. 1976. Congenital glaucoma in the basset hound: a biologic model. Tr. Am. Acad. Ophthalmol. Otol. 81: 645-652. 